Genetic mapping of a gene causing hypertension in the stroke-prone spontaneously hypertensive rat Jacob HJ, et al. Cell 1991;67:213-24.

This paper describes genetic mapping of a gene causing hypertension in an animal model for primary hypertension in humans. Baseline and sodium loaded systolic and diastolic pressures were measured in a cross between the stroke-prone spontaneously hypertensive rat and the normotensive WKY strain. Polymorphic single sequence repeat markers were analysed for linkage to each of these four blood pressure traits. Significant linkage was found between a marker (RD17) on chromosome 10 and both sodium loaded systolic (lod score $=4.88$ ) and diastolic pressures (lod score $=5 \cdot 10$ ). Significant linkage was also observed between a marker on chromosome 18 and baseline diastolic pressure. The locus on chromosome $10(B p 1)$ was shown to be closely linked to the gene encoding angiotensin converting enzyme (ACE) which plays an important role in blood pressure homeostasis and is a target of antihypertensive drugs. This suggests the ACE gene as a possible candidate gene causing hypertension. The identification of the gene at $B p 1$ and other genes involved in hypertension will obviously aid the understanding of the aetiology and pathogenesis of this common disorder. In addition, this study shows the possibility of performing genetic dissection in polygenic quantitative traits related to human disease in the mouse or the rat by crossing inbred strains to eliminate problems of heterogeneity and by using sufficient numbers of animals to achieve the statistical power to detect genes affecting quantitative traits.

\section{N S THAKKER}

\section{Non-paternity and prenatal genetic} screening

MacIntyre S, Sooman A. Lancet

1991;388:869-71.

Prenatal screening programmes focusing on maternal AFP or fetal chromosomal anomalies are independent of paternity. Genetics service laboratories have long recognised the importance of correct paternity in prenatal testing for mendelian disorders. With population screening for cystic fibrosis now being evaluated, instances of wrong paternity are coming to light. Awareness of wrong paternity in population screening programmes is important not only in terms of evaluating costs and benefits of such schemes but, more importantly, in appropriate management where wrong paternity has altered the a priori risk. Estimates as to the true prevalence of wrong paternity are many. This paper evaluates the data from several sources, scientific and popular, whose estimates of wrong paternity range from $1.4 \%$ to $30 \%$. It concludes that reliable data are currently lacking and emphasises the importance of wrong paternity considerations for service planners and service providers in the context of population screening for cystic fibrosis.
A gene deleted in Kallmann's syndrome shares homology with neural cell adhesion and axonal path-finding molecules

Franco B, et al. Nature 1991;353:529-36.

Embryological evidence suggests that the cardinal features of Kallmann's syndrome (hypogonadotrophic hypogonadism and anosmia) are related to a defect of neuronal migration at the olfactory placode. Autosomal dominant, recessive, and $\mathrm{X}$ linked forms of the disease have all been described. Franco et al have now characterised a gene (KALIG-1, in band Xp22.3) that is a strong candidate for the site of the $\mathrm{X}$ linked defect. The methodical collection and mapping of a series of affected patients with terminal $\mathrm{Xp}$ deletions or $\mathrm{X} ; \mathrm{Y}$ translocations enabled localisation of the gene to $\mathrm{a} \sim 80 \mathrm{~kb}$ interval. Making elegant use of standard techniques (identification of $\mathrm{CpG}$ islands, zoo blots, yeast artificial chromosome and cDNA libraries) the authors identified cDNAs within the critical region and constructed a full length sequence from overlapping clones that translates to a protein of 680 amino acids. Three domains, each comprising about 25 amino acids, show significant homology either to protease inhibitors/neurophysins, or to neural cell adhesion molecules/tyrosine phosphases. The latter proteins have been implicated in cell adhesion and neurite outgrowth, supporting the notion that KALIG1 may represent the first neuronal migration factor to be identified in humans. The authors do not explain why it appears to be ubiquitously expressed at low levels, but no doubt they are working on that.

ANDREW WILKIE

The phenylketonuria locus: current knowledge about alleles and mutations of the phenylalanine hydroxylase gene in various populations

Konecki DS, Lichter-Konecki U. Hum Genet 1991;87:377-88.

The screening which detects and the diet which corrects the mental retardation which would otherwise affect the untreated newborn with phenylketonuria is a paradigm of preventive medicine. In this detailed review, the authors summarise the molecular analysis of the mutations at the phenylalanine hydroxylase (PAH) locus on chromosome 12 which underlie the disease phenotypes. Using PAH cDNA as a probe shows eight RFLPs which indicate that specific mutations tend to segregate with specific haplotypes. Thirtyone different DNA alterations have been detected to date; only five are deletions while the remaining 26 are single base substitution leading in most cases to missense mutations. Correlation of mutation, residual activity of the PAH enzyme, and clinical phenotype in dicate that not every homozygous mutation leads to PKU, while the phenotype resulting from the inheritance of two different mutations depends on the residual activity (RA) of each. With an incidence as high as 1 in 16000 in the Chinese, phenylketonuria is not only a Caucasian disease. In Europe there are radical differences between the haplotype frequencies and their associated mutations north and south of the Alps. As with cystic fibrosis, any possible heterozygote advantage remains controversial.
Localisation of the gene encoding the GABAa receptor $\beta 3$ subunit to the Angelman/Prader-Willi region of human chromosome 15

Wagstaff J, Knoll JHN, Fleming J, et al. Am $\mathcal{F}$ Hum Genet 1991;49:330-7.

Prader-Willi syndrome (PWS) and Angelman syndrome (AS) have recently become the focus of considerable interest from clinicians and molecular biologists. These two clinically distinct conditions are associated with cytogenetically similar deletions, 15q11-q13, Prader-Willi critical region (PWCR), and uniparental disomy (UPD). In PWS the deletions are of paternal origin and UPD is maternal disomy, and vice versa in AS. To date no gene of known function had been mapped to the PWCR. In this report, a genomic library was screened with bovine GABAa receptor $\beta 1$ subunit. This identified two clones containing the homologous human GABAa receptor $\beta 3$ subunit. These clones were used as probes and localised by in situ hybridisation to 15q11-q13. Dosage analysis of a panel of cytogenetically deleted PWS and AS patients identified hemizygosity for these two probes. Subsequent study of two subjects with unbalanced translocations, one with PWS and the other with AS, showed that both were hemizygous for the classical markers in the PWCR but that only the AS patient was deleted for the two new probes. It is believed defects in the GABA receptor function might cause seizures, movement disorders, and uncontrollable behaviour; these are the features of AS but not PWS. The possibility that GABR $\beta 3$ might play a role in the aetiology of AS is an exciting step forward and ultimately could help explain the lower frequency of UPD and higher recurrence risk in AS compared to PWS.

T R P COLE

Association of nm23-H1 allelic

deletions with distant metastases in colorectal carcinoma

Cohen KH, Wang F, DeSoto-LaPaix F, et al. Lancet 1991;338:722-4.

Tumour invasion, lymphatic metastases, and distant metastases have a significant inverse association with patient survival in colorectal cancer. This association is inexact and does not allow accurate classification of patients into risk groups, thereby making prognostication difficult in the individual case Cohen $e t$ al have now sought to identify the prognostic significance of alterations at the $\mathrm{nm} 23-\mathrm{H} 1$ locus at $17 \mathrm{q} 21$ in patients with colorectal cancer. The nm23 gene is known to show reduced expression in human breast cancers with nodal metastases. Its possible tumour suppressor role has been further supported by transfection studies. Eleven of the 21 patients entered in this study had deletions with the $\mathrm{nm} 23-\mathrm{H} 1$ probe. These 11 patients had a significantly higher incidence $(p=0.03)$ of distant metastases than had their 10 counterparts who did not show deletion. Does the $\mathrm{nm} 23-\mathrm{H} 1$ locus have a role as a late acting tumour suppressor in colorectal cancer? Have Cohen et al identified a clinically valuable prognostic measure in colorectal cancer? A larger patient group and longer follow up time than the 25 months in this study will be required to answer these pertinent questions. The preliminary data look encouraging. 